\title{
PROGETTARE UN LIBRO DI TESTO DIGITALE TRA CRITICITÀ E OPPORTUNITÀ
}

\section{DESIGNING A DIGITAL BOOK BETWEEN CHALLENGES AND OPPORTUNITIES}

\author{
Andrea Nardi, Dipartimento di Scienze della Formazione e Psicologia \\ (SCIFOPSI), Università degli Studidi Firenze, andrea.nardi@unifi.it
}

\section{SOMMARIO}

In Italia la vigente cornice legislativa prevede nelle scuole la transizione al libro di testo elettronico e individua nei docenti i principali soggetti coinvolti nell'autoproduzione e valutazione di questi materiali didattici digitali. Nel presente studio vengono descritti il design, lo sviluppo e la valutazione di un prototipo di libro di testo digitale, multimediale e interattivo, nel tentativo di indagare le principali criticità poste dalla sua realizzazione, i possibili elementi di innovazione rispetto al tradizionale libro di testo, e fornire delle linee-guida ai docenti sempre più responsabili della scrittura e validazione di queste nuove tipologie di testi. Per valutare l'usabilità cognitivo-didattica del prototipo è stato somministrato un questionario a un panel di esperti. Nella prima parte dell'articolo vengono descritti i risultati della rassegna della letteratura svolta al fine di rintracciare buone regole e principi di design; la seconda parte descrive lo sviluppo del prototipo e la procedura di raccolta e analisi dei dati. I risultati ottenuti vengono successivamente discussi ed infine si cerca di trarre qualche conclusione da quanto emerso.

\section{PAROLE CHIAVE}

Lettura digitale, libro di testo digitale, design, linee guida, ergonomia cognitiva. 


\begin{abstract}
In Italy the current legislative framework foresees the transition to the electronic textbook in schools and identifies teachers as the main subjects involved in the self-production and evaluation of these digital teaching materials. This paper describes the design, development and evaluation of a digital, multimedia and interactive textbook prototype, in an attempt to investigate the main critical issues raised by its implementation and the possible elements of innovation compared to the traditional textbook. In addition, it provides guidelines for teachers who are increasingly responsible for the writing and validation of these new types of texts. To assess the cognitive-didactic usability of the prototype, a questionnaire was given to a panel of experts. The first part of the paper describes the outcomes of the literature review carried out in order to draw effective design principles; the second part illustrates the prototype development and the procedure adopted to collect and analyze data. The results are then discussed and finally some conclusions are drawn.
\end{abstract}

\title{
KEYWORDS
}

Digital reading, e-textbook, design, guidelines, cognitive ergonomics.

Autore per corrispondenza

Andrea Nardi, Dipartimento di Scienze della Formazione e Psicologia (SCIFOPSI), Universitàdegli Studi di Firenze, andrea.nardi@unifi.it 


\section{Introduzione}

Molti Governi stanno già investendo risorse consistenti nell'introduzione dei nuovi dispositivi di lettura e capita sempre più spesso di vedere scuole all'estero, ma anche sul territorio nazionale, in procinto di abbandonare il testo cartaceo per l'adozione di libri di testo digitali.

La riflessione sulla progettazione di libri di testo elettronici è quanto mai attuale anche in Italia dal momento che la vigente cornice legislativa di riferimento prevede adozioni obbligatorie in formato digitale 0 misto $^{1}$ e la possibilità per le scuole di elaborare in proprio i materiali didattici digitali. ${ }^{2}$ Mancano tuttavia ancora modelli e framework condivisi per il design e lo sviluppo dei nuovi testi, così come linee guida per l'inserimento dei nuovi devices nelle pratiche e consuetudini didattiche e sia le case editrici che le singole realtà scolastiche tentano di recepire le indicazioni legislative, mettendo in atto le prime sperimentazioni delle quali andrebbe verificata l'effettiva efficacia.

Il Decreto n. 781 del 2013 invitava a utilizzare «strumenti e standard aperti e interoperabili, nella prospettiva dell'integrazione delle funzionalità di volta in volta implementate all'interno di un framework comune» (MIUR, 2012), di fatto, però, non definiva cosa si dovesse intendere per questa cornice comune né vincolava editori, produttori e docenti all'adozione di soluzioni tecnologiche standardizzate, producendo una forte frammentazione dei materiali didattici realizzati e la loro scarsa interoperabilità.

Un ulteriore errore è forse l'avere dato per scontato da un lato che i docenti possedessero o dovessero possedere le competenze necessarie alla realizzazione dei nuovi testi, senza definire eventuali percorsi formativi e di aggiornamento professionale, dall'altro che le scuole fossero tecnologicamente in grado di sostenere quanto previsto dalla normativa. In pratica si obbligava all'adozione di libri di testo digitali e poi si lasciava la scuola nella condizione di dover reperire le attrezzature e supportare laddove necessario i docenti non preparati all'uso, alla scrittura e alla valutazione dei nuovi testi. Quest'ultima rappresenta sicuramente l'elemento più critico dal momento che l'Articolo 6 della Legge 128 del 2013 affida ai docenti il ruolo di «supervisore della qualità scientifica e didattica» (MIUR, 2013) dei materiali autoprodotti, ma le procedure di validazione non vengono esplicitate, non si capisce se la valutazione debba essere a carico dello stesso docente che ha gestito la produzione, a carico di un docente terzo e nel caso come si debba procedere per individuarlo (Roncaglia, 2018).

Il Ministero ha attuato il passaggio alla versione digitale dei testi con l'obiettivo primario di abbattere i costi di produzione dei materiali didattici (non a caso

\footnotetext{
Versione cartacea più elementi digitali a corredo obbligatori.

2 L'adozione obbligatoria dei libri di testo «nelle versioni on line scaricabili da internet o mista» è stata stabilita dal MIUR attraverso nell'articolo 15 della Legge n. 133 del 2008 recante disposizioni urgenti per lo sviluppo economico, la semplificazione, la competitività, la stabilizzazione della finanza pubblica e la perequazione tributaria.
} 
l'introduzione del libro di testo elettronico è stata inserita in un provvedimento economico). Anche se probabilmente la transizione ai libri di testo digitali non rappresenta l'azione più efficace da mettere in campo per limitare le spese a carico dell'Istituzione scolastica, ${ }^{3}$ in questa sede l'interesse è rivolto alle implicazioni pedagogiche e formative della lettura digitale, ai riflessi sulla didattica, in un momento in cui il dibattito sembra essere invece caratterizzato da istanze che hanno ben poco a che fare con motivazioni educative, ma dove prevalgono aspetti pratici, questioni commerciali e di diritto d'autore, di proprietà delle tecnologie coinvolte, performance dei vari devices (Calvani, 2013). Qui interessa l'aspetto didattico/pedagogico; in particolare, si intende indagare se l'utilizzo di un libro di testo digitale, al di là di novità, mode e «appeal» del momento, possa rappresentare un reale valore aggiunto, e quando, per i processi di apprendimento. L'attenzione sarà quindi rivolta all'ergonomia cognitiva, all'usabilità didattica, al design, alle affordances e alle features dell'e-textbook, punti di forza per dare al libro di testo digitale una collocazione didatticamente utile.

Il focus di questa riflessione è il libro di testo inteso come oggetto di studio, un prodotto che esalti l'operazione di studio individuale e in auto-apprendimento. Verranno quindi presi in considerazione i cosiddetti e-textbook, vale a dire libri di testo elettronici specificatamente progettati per l'apprendimento, per differenziarsi nettamente da altre tipologie di testo (e-book narrativi, magazine elettronici, quotidiani digitali, moduli e ipertesti navigabili online). Si intende qui per e-textbook un libro, in forma digitale arricchita, ma che mantenga la sua «forma libro» e quindi una determinata struttura e specifiche affordances che permettano la comprensione in profondità delle informazioni. Il manuale scolastico rappresenta infatti un modello di riferimento condiviso di strutturazione del sapere, che abitua la mente di chi apprende a una modalità specifica di organizzazione delle informazioni che condiziona profondamente i processi di apprendimento. Da questo punto di vista è vero probabilmente che ad oggi gli esempi «più interessanti di e-book arricchiti siano proprio quelli che riescono a unire l'innovazione rappresentata da contenuti interattivi e multimediali alle caratteristiche di autoconsistenza, autorialità forte e articolazione complessa ma fondamentalmente lineare proprie della forma-libro» (Roncaglia, 2013, p. 6).

Già da qualche anno si stanno sperimentando queste nuove tipologie di testo scolastico, ma nella maggior parte dei casi i prodotti realizzati risultano ancora poco più di semplici conversioni del formato cartaceo in formato digitale, senza che sia così possibile valutare gli effetti - e i possibili elementi di innovazione - di alcune peculiarità del testo digitale. Il libro di testo digitale ha però senso soltanto se arricchisce e offre vantaggi dal punto di vista degli apprendimenti rispetto alla

3 Pensiamo, ad esempio, al dispendio in termini di acquisizione delle competenze necessarie allo sviluppo multimediale, ai costi necessari all'acquisto dei dispositivi di lettura, dei software di elaborazione grafica, montaggio e programmazione, a quelli connessi alla gestione e al mantenimento delle piattaforme di raccolta e condivisione dei materiali; a quelli derivanti dall'utilizzo di materiale coperto da copyright. 
controparte cartacea. Tra le opportunità offerte dalla lettura di un testo digitale, tre elementi sembrano emergere con più forza: (i) l'ipertestualità, cioè la possibilità di navigare attraverso link predisposti e di arricchire il testo con risorse e contenuti di approfondimento; (ii) l'interattività, qualora sia possibile ottenere informazioni aggiuntive dal testo e/o un feedback sul proprio apprendimento, (iii) la multimedialità, cioè il fatto che, al di là dei codici consentiti sulla carta, si possa usufruire di più linguaggi come ad esempio l'immagine dinamica e il suono. Ci si dovrebbe chiedere in quali circostanze concrete ciascuna di queste caratteristiche possa diventare pedagogicamente rilevante. Questo studio intende fornire non soltanto delle possibili linee-guida per gli insegnanti sempre più coinvolti e responsabili del processo di scrittura e valutazione dei prodotti, ma anche alcune vie percorribili per la realizzazione in un'ottica più ampia di policy dell'editoria.

\section{Linee guida, buoni principi e regole di design}

Lo scopo della seguente rassegna della letteratura è quello di fare il punto su quanto emerge dall'analisi degli studi, delle ricerche e degli esperimenti che hanno valutato potenzialità e criticità del processo di realizzazione di libri di testo digitali al fine di:

- indagare alcuni aspetti di ergonomia cognitiva e didattica del libro di testo digitale;

- trovare, se disponibili, linee guida e standard condivisi di progettazione e sviluppo;

- rintracciare buoni principi e regole di design;

- fornire indicazioni utili allo sviluppo di un libro di testo digitale.

\subsection{EBONI (Electronic Books ON-screen Interface)}

Particolarmente utili alla progettazione e realizzazione di libri di testo digitali, anche se un po' datati, risultano i lavori sviluppati all'interno del progetto EBONI (Electronic Books ON-screen Interface) e quanto fatto negli ultimi anni dal gruppo di ricerca dell'Università della Svizzera Italiana, coordinato da Monica Landoni. Un primo studio denominato Visual Book (Landoni, 1997) ha ipotizzato che mantenere anche per le pubblicazioni elettroniche la stessa struttura e la stessa interfaccia dei testi a stampa potesse facilitarne l'accesso e la fruizione da parte dell'utente, e concluso che il modello libro gioca un ruolo di primaria importanza anche per la progettazione di e-book (Landoni, Crestani \& Melucci, 2000; Landoni, 1997). Successivamente lo studio «Web Book» (Landoni, Wilson \& Gibb, 2000) ha valutato se l'usabilità del testo elettronico potesse essere incrementata dal cambiamento della presentazione del contenuto, adattandosi alle caratteristiche del nuovo tipo di supporto (Crestani, Landoni \& Melucci, 2005). Le Electronic Textbook Design Guidelines (Wilson, Landoni, \& Gibb, 2002), nate dall'esperienza di queste due ricerche, indagano prevalentemente il problema dell'usabilità dell'interfaccia dei 
libri elettronici e raccolgono una serie di raccomandazioni utili alla loro realizzazione; vediamo ed integriamo alcune di queste. ${ }^{4}$

- Copertina. Anche il libro elettronico dovrebbe avere una copertina perché grazie alla sua presenza il lettore ha la percezione di stare per leggere una serie di pagine che fanno parte di un insieme e che possono essere rilette o recuperate.

- Metafora del libro cartaceo. Il libro digitale dovrebbe assomigliare, essere coerente e rispecchiare il modello libro senza creare ambiguità e conflitti. I lettori si avvicinano infatti ai testi in formato elettronico, con le aspettative ereditate dalla loro esperienza sui libri di carta e rimangono per questo spesso delusi. Il look and feel del libro elettronico (Wilson, 2003) gioca un ruolo cruciale nella fruizione del nuovo formato: «Le persone cercano di adattarsi all'esperienza della lettura digitale in modelli mentali derivanti dalla cultura della stampa [...] il modo in cui gli utenti comprendono e descrivono le loro esperienze di lettura sui dispositivi digitali sono modellate dalle aspettative culturali ben consolidate riguardo al libro stampato» (MacFadyen, 2011, pp. 2-3). Strutture e layout simili tra libri di testo digitali e cartacei aiutano gli studenti andando incontro alle loro abitudini (Gong, Chen, Wang, Zhang \& Huang, 2013). ${ }^{5}$ Mostrare i contenuti di un libro elettronico su pagine strutturate sul modello che richiama e assomiglia a quello del libro fisico aumenta il confort dell'utente e garantisce una struttura logica riconoscibile che permette al lettore di acquisire adeguatamente le informazioni.

- Collegamenti. Quando leggiamo un libro elettronico un numero eccessivo di collegamenti ipertestuali che rimandano a informazioni aggiuntive rischiano di creare un senso di non appartenenza e di disorientamento nel lettore. Dovrebbero per questo essere chiare e visibili indicazioni e funzioni che permettano all'utente di non «perdersi» e diano la stessa sensazione di spazio che fornisce il libro tradizionale. I lettori si aspettano sostanzialmente un sistema che, in qualche modo, simuli e integri le possibilità di ricerca che già sfruttano per i testi a stampa: il rinvio tra le pagine di un libro, tra il testo e le note, tra due o più testi, sono da sempre una prerogativa dei libri scolastici (e non solo). La lettura di ipertesti pone inoltre un carico cognitivo aggiuntivo al lettore cliccare e scorrere il testo, valutare continuamente i link, fare scelte di navigazione, elaborare una molteplicità di stimoli sensoriali - e questo sovraccarico cognitivo può ostacolare le prestazioni di lettura e la comprensione del testo (DeStefano \& LeFevre, 2007). Occorre quindi fare un uso moderato dell'i-

4 Per ulteriori indicazioni un buon riferimento è la Guide de conception et d'utilisation du manuel numérique universitaire realizzata dall'Università del Québec che sempre a partire dalle indicazioni dell'esperienza EBONI ne ha attualizzato i suggerimenti concentrandosi sul target universitario. La guida è scaricabile al seguente indirizzo: https://bit.ly/2uzRFbu

5 Jakob Nielsen suggerisce tuttavia che, per avere successo, un testo elettronico non debba semplicemente imitare la sua controparte cartacea. Si ritiene che il nuovo mezzo coinvolga il lettore in modo diverso e che esperienze più potenti possano essere raggiunte deviando dal flusso lineare del testo (Nielsen, 2008). 
pertestualità, segnalare adeguatamente i rimandi online e prevedere sempre percorsi per tornare in modo semplice al punto di partenza.

- Tavola dei contenuti o Indice. Altre importanti caratteristiche del libro che dovrebbero essere mantenute nel formato elettronico sono la tavola dei contenuti e l'indice, strumenti fondamentali che permettono all'utente di farsi un'idea sul materiale che dovrà leggere e che non devono essere sostituiti semplicemente con funzioni di ricerca, ma sfruttando i collegamenti intertestuali tra gli argomenti indicizzati e le relative sezioni del testo. Questi strumenti di navigazione in un e-book divengono ancora più importanti se si pensa che il lettore non ha la possibilità di monitorare la propria posizione nel testo confrontando lo spessore e il peso delle pagine già lette con quelle ancora da leggere, e non può contare su ancoraggi spaziali e importanti elementi informativi di contesto presenti invece nei libri cartacei, che guidano e orientano chi legge, e che nel digitale si perdono (Li, Chen \& Yang, 2013).

- Lunghezza delle pagine. Pagine troppo lunghe o, al contrario, troppo corte rendono la fruizione del testo elettronico difficoltosa. La pagina dovrebbe essere trattata come uno spazio visivo in cui le informazioni possono essere facilmente reperite e scansionate. Una migliore consultazione può essere garantita dividendo la pagina in piccoli blocchi, per esempio alternando immagini a diagrammi o inserendo titoli e sottotitoli. Studi di eye-tracking hanno infatti verificato che quando una pagina digitale è suddivisa in chiari e ben visibili blocchi di testo da intestazioni, punti, elenchi numerati, titoli e sottotitoli più grandi, in grassetto, di un colore diverso, di un diverso carattere, aumenta la sua leggibilità, fornisce adeguati percorsi di lettura, evitando l'attivazione di errati modelli di scansione delle informazioni che portano spesso i lettori a saltare contenuti importanti semplicemente perché questi non risultano adeguatamente formattati per la lettura su schermo (Pernice, 2017).

- Uso dei colori. Troppi colori rischiano di distrarre e uno sfondo troppo bianco (specialmente nel caso di e-reader con schermo retroilluminato) potrebbe dare fastidio agli occhi. L'ideale è sfruttare background il più possibile neutri.

- Leggibilità. È necessario curare con attenzione gli elementi visivi a supporto del testo (video, immagini, icone e bottoni, tabelle, grafici, infografiche), gli aspetti tipografici, pensando alla giusta collocazione degli elementi multimediali e di navigazione, alla quantità di testo da assegnare ad ogni pagina, per migliorare la leggibilità. ${ }^{6}$

\footnotetext{
Per reperire questo tipo di materiale il sito Creative Commons Image Search (https://search. creativecommons.org/) permette di fare ricerche ottenendo i risultati forniti da bacini come $E u$ ropeana e Flickr, filtrando i risultati in base a varie modalità di riuso. L'Internet Archive (https:// archive.org/) contiene materiale multimediale di pubblico dominio. Per reperire immagini in alta definizione ci sono servizi come Unsplash (https://unsplash.com/) dove comunità di fotografi offrono gratuitamente i propri scatti. Per icone libere da copyright si possono consultare siti come The Noun Project (https://thenounproject.com/). Per quanto riguarda infine i fonts è possibile scegliere caratteri pensati e progettati per la lettura su schermo come Literata (https:// www.type-together.com/literata) e Bookerly (https://en.wikipedia.org/wiki/Bookerly).
} 
- Indizi visivi. Gli indizi visivi, come ad esempio le icone, devono essere adattati per sfruttare le potenzialità del mezzo elettronico.

\subsection{Ergonomia cognitiva e didattica}

Alcuni principi ergonomico-cognitivi rintracciati in letteratura risultano essenziali alla progettazione di libri di testo digitali. Tra i concetti più importanti nel campo dell'ergonomia cognitiva c'è quello di usabilità di un sistema. Jakob Nielsen (1993) raccomanda dieci caratteristiche che permettono di valutare il grado di l'usabilità e che andrebbero tenute in considerazione dal momento che i libri di testo digitali rappresentano a tutti gli effetti interfacce di lettura (Roncaglia, 2010). L'interfaccia dovrebbe andare incontro alle necessità dei fruitori e riflettere il modello mentale con il quale si approcciano allo specifico strumento, che cosa si attendono dal sistema, quindi le attese, sia esplicite che implicite. Ad esempio in un ipertesto è bene sottolineare le parole che sono collegamenti, perché gli utenti sono oramai abituati a questa convenzione ed è divenuta un invito all'uso.

Un concetto fondamentale per il design delle interfacce è quello di affordance, termine divenuto negli ultimi anni sempre più popolare anche nel campo della lettura digitale (Railean, 2015). Le affordances offerte dall'e-texbook non dipendono soltanto dalla relazione tra discente e il contenuto ma anche dal tipo di apprendimento che viene attivato e permesso da questa relazione, ovvero dal «learning design» del libro di testo digitale. In quest'ottica altre indicazioni utili per la progettazione di testi interattivi e multimediali provengono da quel filone di studi che va sotto il nome di Interaction Design (ID), una disciplina della progettazione meccanica che si focalizza sull'interazione uomo-macchina (Dix, Finlay, Abowd \& Beale, 2004). L'interaction design possiede diversi strumenti e metodi che possono facilitare la progettazione di libri elettronici: Le dieci euristiche di Nielsen (1993); I sette principi dialogici ISO 9241-110 (2006); I principi di design per l'usabilità di Dix e colleghi (2004); Le otto regole d'oro (golden rules) del design di Shneiderman \& Plaisant (2009); I criteri ergonomici di Bastien e Scapin (1993; 1997).

Il libro di testo digitale si pone al crocevia tra gli strumenti didattici tradizionali e le nuove tecnologie: da una parte mantiene alcuni aspetti specifici dell'interfaccia del libro cartaceo, dall'altro assume nuove configurazioni grazie agli sviluppi tecnologici. La riflessione sul libro di testo elettronico va quindi collocata inevitabilmente al centro della riflessione più ampia sulla cosiddetta «ergonomia didattica» (Calvani, 2002) degli strumenti tecnologici per l'apprendimento. Il problema da risolvere deve essere tale da giustificare l'impiego del mezzo per la sua risoluzione (e-book, online vs libro cartaceo). Il rapporto con il mezzo non deve essere tale da assorbire di per sé troppa energia a scapito della soluzione del problema (attrazione dell'interfaccia e piacere di manipolare). Il soggetto non deve essere indotto a delegare le funzioni interne allo strumento (delega e disabilitazione cognitiva). Si devono evitare fattori di dispersione dell'attenzione e sovraccarico 
(attenzione divisa vs lettura profonda), così come eccessiva discarica cognitiva (eccessivo alleggerimento del carico cognitivo vs impegno nella risoluzione di problemi complessi). L'elevato livello di coinvolgimento prodotto dall'interazione con il medium elettronico di lettura non deve compromettere la riflessività dal momento che il primo richiede un coinvolgimento diretto, mentre la seconda un distaccamento (interattività vs riflessività) (Calvani, 2008).

Sembra quindi che alcune caratteristiche dei testi digitali come la possibilità di integrare simulazioni interattive e dinamiche siano particolarmente funzionali alle discipline scientifiche, mentre questo è forse meno vero nel caso della lettura di un lungo trattato filosofico, dove la capacità di comprendere le complesse questioni risulta proporzionale alla possibilità di mettere in atto una lettura quanto più possibile silenziosa, concentrata, attenta e profonda. In quest'ottica non esiste probabilmente un unico modello di riferimento per il manuale digitale, ma una serie di modelli possibili, in base all'uso e alla disciplina di riferimento.

\subsection{Multimedia Cognitive Learning Theory e Cognitive Load Theory}

Secondo Thierry Baccino, professore presso l'Università Paris VIII in Psychologie Cognitive des Technologies Numériques, la progettazione di documenti elettronici dovrebbe seguire il più possibile il concetto di «intuitivité perceptive» (Baccino \& Drai-Zerbib, 2012). L'intuitività percettiva può essere declinata sulla modalità di percezione (organizzazione percettiva delle informazioni, affordance) o cognitiva (la familiarità delle situazioni riscontrate) ed è dominata da processi per lo più inconsci che guidano la nostra visione e il modo in cui il cervello costruisce un'immagine della realtà. ${ }^{7}$ Ad esempio l'organizzazione degli elementi testuali e non testuali dovrebbe permettere in primo luogo di identificare gli elementi simili e riconoscere in modo rapido ed efficiente una scena visiva. Il raggruppamento dei blocchi informativi della stessa dimensione di una pagina porta ad una migliore identificazione delle informazioni ed è per questo che il principio di similitudine viene generalmente rispettato nell'ergonomia dell'interfaccia (Baccino \& Drai-Zerbib, 2012).

Per indagare questi meccanismi cognitivi abbiamo oggi le evidenze sperimentali prodotte dalla teoria cognitiva dell'apprendimento multimediale (Multimedia Cognitive Learning Theory) e dalla teoria del carico cognitivo (Cognitive Load Theory) che, anche se indirettamente, forniscono indicazioni utili alla progettazione e alla scrittura di libri di testo elettronici. Anche quest'ultima infatti dovrebbe seguire alcuni principi di principi di comunicazione visiva e multimediale in modo da evitare fenomeni di sovraccarico cognitivo, mantenendo sempre una coerenza tra i diversi codici utilizzati e non interferendo con la com-

\footnotetext{
Alcuni di questi meccanismi sono stati identificati fin dall'inizio del ventesimo secolo dagli psicologi della Gestalt, e si basano sulla capacità dell'occhio umano di distinguere i vari elementi di una scena visiva raggruppando o distinguendoli secondo una serie di principi percettivi fondamentali: somiglianza, chiusura, continuità e prossimità (Wertheimer, 1923).
} 
prensione del testo. Queste due teorie hanno mostrato come l'inserimento in un testo digitale di elementi multimediali ed ipertestuali - se non correttamente eseguito - possa avere un impatto negativo in termini di apprendimento. L'uso della multimedialità incontrollata, i dettagli seducenti, gli elementi a scopo puramente «decorativo», l'immagine seduttiva, anche se catturano l'attenzione non la orientano verso gli elementi significativi e possono produrre fenomeni di sovraccarico cognitivo e attenzione divisa, interferendo con il compito di apprendimento (Clark \& Lyons, 2011). La scelta, selezione e posizionamento degli elementi a supporto del testo dovrebbero quindi seguire alcuni principi:

- Vicinanza/contiguità spaziale. Il principio di contiguità raccomanda che contenuti correlati debbano essere inseriti in pagine o schermate in modo integrato: è stato infatti dimostrato che immagini e parole separate rischiano di deprimere l'apprendimento rispetto alle stesse immagini e parole presentate in modo integrato (Sweller, van Merrienboer \& Paas, 1998). Studi di eyetracking hanno confermato questi dati mostrando differenze di distribuzione dell'attenzione durante la lettura di testi con elementi visivi separati o integrati nel testo (Holsanova, Holmberg \& Holmqvist, 2009): nel primo caso i soggetti esaminati trattano le immagini e il testo come due unità indipendenti di informazione, al contrario, nel testo integrato i lettori esaminano le immagini e il testo come un tutt'uno.

- Modalità. Riguarda il principio secondo il quale in parte si può ottimizzare l'impiego cognitivo ovviando ai limiti della memoria di lavoro se si distribuisce il carico su due canali distinti (uditivo e visivo): ad esempio la presentazione di un grafico accompagnato da un audio sembra funzionare meglio dello stesso grafico accompagnato da una descrizione scritta (Moreno \& Mayer, 1999).

- Ridondanza. Secondo questo principio un contenuto che duplica un altro contenuto anziché aiutare ostacola l'apprendimento. A volte una sola fonte di informazione è più efficace e comprensibile perché non aggiunge informazione non rilevante o ridondante e non va a incidere negativamente sul carico cognitivo. La presentazione di molteplici fonti di informazione, ciascuna delle quali può essere compresa isolatamente, può sovraccaricare la memoria di lavoro e produrre peggiori risultati di apprendimento (Sweller et al., 1998).

- Capovolgimento dell'expertise. Diversi esperimenti hanno dimostrato differenze legate al livello di conoscenza pregressa: un supporto aggiuntivo utile per i novizi può diventare all'opposto poco efficace o anche ridondante (e quindi dannoso) per gli esperti. Ad esempio gli elementi visivi aggiunti al testo migliorerebbero notevolmente l'apprendimento dei novizi, ma non degli esperti (Kalyuga \& Renk1, 2010).

Se dovessimo tentare di applicare i principi della teoria cognitiva dell'apprendimento multimediale e della teoria del carico cognitivo alla progettazione di un libro di testo efficace quest'ultimo dovrebbe: evitare stimoli seduttivi e decorativi non funzionali al compito di apprendimento; fornire il giusto grado di libertà e sco- 
perta, un feedback costante e immediato, non attivare errate preconoscenze, evitare fenomeni di sovraccarico, dispersione cognitiva e attenzione divisa, fare un buon utilizzo di indizi che riducano il carico cognitivo, e rispettare il grado di expertise del lettore. Si capisce bene quanto possa essere complesso il processo di design di un materiale didattico di questo tipo, e quanto le cose si complichino nel momento in cui si intenda arricchirlo con elementi multimediali, interattivi e ipertestuali.

\section{Contesto e procedura dello studio di prototipazione}

\subsection{Obiettivo della ricerca}

Obiettivo di questo lavoro è quello di individuare quali possano essere i principi da seguire per una buona progettazione/sviluppo di libri di testo elettronici, e indagare se alcune prerogative e peculiarità del digitale possano rappresentare elementi di valore aggiunto rispetto al tradizionale libro di testo stampato.

\subsection{Ipotesi di ricerca}

Abbiamo ipotizzato che il testo digitale abbia alcune potenzialità che possono rappresentare un valore aggiunto in termini di apprendimento, così come alcuni elementi di criticità che tuttavia possono esseri tenuti sotto controllo in fase di progettazione, in modo da creare testi digitali efficaci. Alcune indagini indicano infatti che se le funzionalità elettroniche dell'e-book sono ben progettate, i risultati e l'atteggiamento degli utenti verso i libri di testo digitali tende a migliorare (Gu, Wu \& Xu, 2014). Per testare la nostra ipotesi abbiamo costruito un prototipo, un «testo ideale», in base alle indicazioni della letteratura. La prototipazione ha visto lo sviluppo di una versione multimediale e interattiva del testo d'esame: Ranieri, M. (2011). Le insidie dell'ovvio. Tecnologie educative e critica della retorica tecnocentrica. Pisa: ETS, utilizzato presso l'Università degli Studi di Firenze, Dipartimento di Scienze Formazione e Psicologia, dagli studenti che hanno frequentato il corso di «Nuove Tecnologie per l'Educazione e la Formazione» e «Tecnologie dell'Istruzione e dell'Apprendimento». Le affordances del prototipo sono state pensate soprattutto in rapporto ai contenuti e a una loro migliore comprensione. Le domande che di volta in volta ci siamo posti durante il processo di progettazione sono state: qual è in questo specifico caso il vantaggio dell'uso di un e-book rispetto al libro cartaceo? Come posso facilitare la comprensione di questo concetto?

\subsection{Campione}

La valutazione/validazione del prototipo è stata assegnata a un panel di dieci esperti. Il panel è stato selezionato secondo un criterio di convenienza, coinvolgendo studiosi di lettura digitale, editoria, tecnologie didattiche e comunicazione 
digitale. È infatti generalmente riconosciuto che, quando si tratta di valutare l'usabilità di un prototipo, gli utenti «esperti» impegnati in un test di usabilità siano capaci di rintracciare più errori (Nielsen, 1993). A ogni esperto è stato chiesto di utilizzare il libro per un periodo di circa un mese e compilare un questionario di valutazione dell'usabilità cognitivo-didattica costruito con Google Moduli e somministrato online. È stato fornito un breve tutorial per lo scaricamento del prototipo e la compilazione del questionario. In base alle indicazioni emerse dalla fase valutativa abbiamo riprogettato l'e-textbook cercando di intervenire sugli elementi di maggiore criticità. In Tabella 1 sono riportate le caratteristiche dei partecipanti.

TABELLA 1

Caratteristiche demografiche dei partecipanti $(n=10)$

\begin{tabular}{|c|l|l|}
\hline \multirow{4}{*}{ Genere } & Maschi & 7 \\
\cline { 2 - 3 } & Femmine & 3 \\
\hline \multirow{4}{*}{$\begin{array}{c}\text { Professione } \\
\text { Interessi di } \\
\text { ricerca }\end{array}$} & Professore universitario & 5 \\
\cline { 2 - 3 } & Ricercatore universitario & 2 \\
\cline { 2 - 3 } & Consulente aziendale & 3 \\
\hline \multirow{4}{*}{} & Didattica e comunicazione digitale/multimediale & 10 \\
\cline { 2 - 3 } & Eecnologie educative & 10 \\
\cline { 2 - 3 } & Eedia education/Digital literacy & 8 \\
\cline { 2 - 3 } & Design interfacce/Ergonomia cognitiva & 6 \\
\cline { 2 - 3 } & Editori digitale & 5 \\
\cline { 2 - 3 } & Mobile learning/videoeducazione & 4 \\
\hline
\end{tabular}

\subsection{Criteri e strumenti valutativi}

Il questionario di valutazione è stato realizzato incrociando e selezionando gli elementi più significativi e funzionali alla nostra analisi che provenivano dai seguenti strumenti valutativi: i) le Usability Heuristics for User Interface Design (Nielsen, 1993); ii) gli Evaluation criteria for educational e-books (Díaz, 2003); e iii) gli Evaluation Criteria for Interactive E-Books (Bozkurt \& Bozkaya, 2015). A partire da queste indicazioni è stato costruito un questionario a risposte chiuse composto da 23 quesiti così strutturato:

- Criteri di valutazione dell'usabilità dell'interfaccia: 12 item su elementi come ad esempio la facilità d'uso, l'organizzazione dello spazio, l'impaginazione, il font, l'uso dei colori e di elementi tipografici, l'appropriatezza al target, gli indizi di interfaccia e il sistema di navigazione, il livello di connettività e la prevedibilità del sistema.

- Criteri di valutazione dell'efficacia del supporto per l'apprendimento: 11 item su elementi come ad esempio il layout e la densità semantica, le learning activities, il livello di personalizzazione (annotazioni, appunti, evidenziazioni, 
segnalibri) e approfondimento dei contenuti, l'attrattività del contenuto, il livello di feedback e facilitazione, la coerenza ed efficacia di elementi multimediali ed interattivi.

- Commenti liberi: consigli e indicazioni utili a migliorare il prototipo.

Per la valutazione è stata scelta una scala che va da giudizi molto positivi a non positivi («Sempre» - «Spesso» - «A volte» - «Mai»). La versione integrale del questionario, utile alla valutazione di libri di testo digitali, è riportata in Tabella 2.

TABELLA 2

\section{Questionario di valutazione del prototipo}

\begin{tabular}{|c|c|c|c|}
\hline \multicolumn{3}{|c|}{ Variabile } & Item \\
\hline \multicolumn{4}{|c|}{ Criteri per valutare l'usabilità dell'interfaccia } \\
\hline 1 & Facilità d'uso & \multicolumn{2}{|c|}{ L'interfaccia è facile da usare } \\
\hline 2 & $\begin{array}{l}\text { Organizzazione } \\
\text { dello spazio }\end{array}$ & \multicolumn{2}{|c|}{$\begin{array}{l}\text { L'impaginazione e l'organizzazione dello spazio della pagina sono funzionali } \\
\text { a migliorare la leggibilità? }\end{array}$} \\
\hline 3 & Aspetti tipografici & \multicolumn{2}{|c|}{$\begin{array}{l}\text { Il font, l'uso dei colori e gli elementi tipografici sono funzionali a migliorare } \\
\text { la leggibilità? }\end{array}$} \\
\hline 4 & Appropriatezza & \multicolumn{2}{|c|}{ L'interfaccia ha il look-and-feel appropriato per il target universitario? } \\
\hline 5 & $\begin{array}{l}\text { Caratteristiche } \\
\text { dell'utente }\end{array}$ & \multicolumn{2}{|c|}{$\begin{array}{l}\text { La progettazione dei contenuti tiene conto delle particolari caratteristiche } \\
\text { degli utenti? }\end{array}$} \\
\hline 6 & $\begin{array}{l}\text { Sistema di } \\
\text { navigazione }\end{array}$ & \multicolumn{2}{|c|}{$\begin{array}{l}\text { Ci sono forme multiple di navigazione (navigazione per mezzo di tabelle di } \\
\text { contenuto, indice analitico, ecc.)? }\end{array}$} \\
\hline 7 & Disorientamento & \multicolumn{2}{|c|}{$\begin{array}{l}\text { Il sistema di navigazione è tale da evitare il disorientamento e fornire un } \\
\text { rapido accesso alle informazioni? }\end{array}$} \\
\hline 8 & $\begin{array}{l}\text { Supporto alla } \\
\text { navigazione }\end{array}$ & \multicolumn{2}{|c|}{$\begin{array}{l}\text { Le funzioni di supporto alla navigazione consentono all'utente di } \\
\text { individuare, in qualsiasi momento, la sua posizione nel prodotto? }\end{array}$} \\
\hline 9 & Indizi di interfaccia & \multicolumn{2}{|c|}{$\begin{array}{l}\text { Gli indizi di interfaccia (colore, sfondo, icone) sono applicati coerentemente } \\
\text { in tutte le pagine in modo che l'utente sia in grado di prevedere che cosa } \\
\text { fare quando si trova in un posto diverso del testo? }\end{array}$} \\
\hline 10 & Prevedibilità & \multicolumn{2}{|c|}{$\begin{array}{l}\text { Il sistema è prevedibile? Gli utenti possono anticipare come si comporterà } \\
\text { in risposta ad una loro specifica interazione? }\end{array}$} \\
\hline 11 & Connettività & \multicolumn{2}{|c|}{$\begin{array}{l}\text { L'ipertesto è collegato in modo efficace ed esistono hub e percorsi per } \\
\text { raggiungere tutte le pagine del libro elettronico e viceversa? (es. indici, } \\
\text { sommari). }\end{array}$} \\
\hline 12 & Interazione & \multicolumn{2}{|c|}{$\begin{array}{l}\text { L'interfaccia offre percorsi di navigazione flessibili? (gli utenti possono } \\
\text { leggere un nodo informativo e interagire con esso ma una volta finito } \\
\text { possono decidere di tornare al nodo di input). }\end{array}$} \\
\hline \multicolumn{4}{|c|}{ Criteri per valutare l'efficacia del supporto per l'apprendimento } \\
\hline 1 & Layout & \multicolumn{2}{|c|}{ Il layout è funzionale all'apprendimento dell'argomento trattato? } \\
\hline 2 & Densità Semantica & \multicolumn{2}{|c|}{$\begin{array}{l}\text { L'utilizzo di codici e linguaggi diversi e i conseguenti livelli di densità } \\
\text { semantica ed informativa, sono adeguati all'argomento trattato e agli } \\
\text { obiettivi di apprendimento? }\end{array}$} \\
\hline 3 & $\begin{array}{l}\text { Attività di } \\
\text { apprendimento }\end{array}$ & \multicolumn{2}{|c|}{$\begin{array}{l}\text { Sono previsti compiti in linea con gli obiettivi di apprendimento degli } \\
\text { studenti? (es. la risoluzione di problemi, l'autovalutazione). }\end{array}$} \\
\hline 4 & $\begin{array}{l}\text { Supporto di } \\
\text { Authoring }\end{array}$ & \multicolumn{2}{|c|}{$\begin{array}{l}\text { Il sistema può essere modificato dai lettori? (annotazioni, appunti, } \\
\text { evidenziazioni, segnalibri). }\end{array}$} \\
\hline
\end{tabular}




\begin{tabular}{|c|c|l|}
\hline 5 & $\begin{array}{c}\text { Attrattività e } \\
\text { coinvolgimento }\end{array}$ & Il contenuto risulta attraente e coinvolgente? \\
\hline 6 & Feedback & $\begin{array}{l}\text { L'interfaccia è progettata per aiutare gli studenti a scoprire i progressi fatti, } \\
\text { gli errori e le incomprensioni? }\end{array}$ \\
\hline 7 & Approfondimenti & Il sistema prevede un adeguato volume di approfondimenti? \\
\hline 8 & Facilitatori & $\begin{array}{l}\text { Le facilitazioni fornite (verifiche, concetti chiave, sistema di ricerca, } \\
\text { approfondimenti istantanei, rinforzo tramite codici comunicativi diversi) } \\
\text { sono tali da fornire una facilitazione cognitiva e supportare il processo di } \\
\text { apprendimento? }\end{array}$ \\
\hline 9 & Multimedialità & $\begin{array}{l}\text { L'utilizzo di elementi extratestuali rispetta i principi basilari della teoria } \\
\text { cognitiva dell'apprendimento multimediale (es. contiguità)? }\end{array}$ \\
\hline 10 & $\begin{array}{c}\text { Sovraccarico } \\
\text { Cognitivo }\end{array}$ & $\begin{array}{l}\text { L'utilizzo di elementi extratestuali è tale da non creare fenomeni di } \\
\text { attenzione divisa, dispersione dell'attenzione e sovraccarico cognitivo? }\end{array}$ \\
\hline 11 & Valore aggiunto & $\begin{array}{l}\text { L'utilizzo di elementi extratestuali rappresenta un valore aggiunto per } \\
\text { l'apprendimento se comparato con la versione cartacea del testo? }\end{array}$ \\
\hline
\end{tabular}

\subsection{Elaborazione dei dati}

I dati di tipo quantitativo sono stati analizzati al computer con il programma SPSS; dopo essere stati inseriti in una matrice è stata verificata la presenza di eventuali errori di immissione tramite il controllo casuale di alcuni questionari. I dati quantitativi provenienti dalla valutazione del prototipo sono stati arricchiti con dati di carattere qualitativo, prevenienti dai commenti liberi inseriti dagli esperti al termine del questionario, e dagli scambi intercorsi via mail con il panel durante e a conclusione dello studio. Per le domande a risposta chiusa è stata realizzata un'analisi descrittiva delle frequenze di distribuzione delle risposte, mentre i commenti, suggerimenti e le indicazioni fornite dagli esperti sono stati analizzati per individuare temi e concetti ricorrenti. Una volta stabiliti i temi, si è iniziato a cercare le relazioni reciproche. Lavorando alle possibili connessioni si è giunti infine a quattro macro-categorie nelle quali sono stati sintetizzati i risultati ottenuti: i) potenzialità, ii) criticità, iii) modifiche da apportare al prototipo, iv) possibili sviluppi futuri.

\subsection{Caratteristiche e funzionalità del prototipo}

Per la scrittura del nostro prototipo, dopo una prima fase di ricognizione degli strumenti di authoring, abbiamo deciso optato per il software Ibooks Author di Apple. Il software è risultato il più adatto all'integrazione di elementi multimediali e interattivi e permette la fruizione su supporti di lettura come iPad, con i quali gli studenti hanno buona familiarità. Tra gli svantaggi dell'utilizzo di questo software ci sono l'incompatibilità del prodotto finito con dispositivi non Apple (il software genera infatti un file .iba fruibile soltanto con questa tipologia di strumenti) e la ridotta accessibilità rispetto al formato ePub. Ibooks Author, dalla versione 2.4.1 rilasciata a giugno del 2015, permette di lavorare anche in formato ePub, modalità che però risulta ancora «povera» dal punto di vista degli elementi di interattività integrabili al testo e meno user friendly per quanto riguarda l'impaginazione. Ai 
fini della nostra ricerca abbiamo pertanto ritenuto necessario non lavorare in questa modalità in modo da testare in modo più adeguato le nostre ipotesi.

Nella progettazione del prototipo abbiamo seguito le raccomandazioni che provengono dalla ricerca e dal quadro teorico di riferimento. Abbiamo cercato di attenerci il più possibile alle indicazioni che vengono date nei vari checkpoints presenti nelle linee-guida EBONI e che già si rifanno agli standard e alle raccomandazioni contenute nelle Web Content Accessibility Guidelines prodotte dal World Wide Web Consortium (W3C). In questa sede, per limiti di spazio, riportiamo in Figura 1, 2 e 3 alcuni esempi di funzionalità di funzionalità, rimandando online (http:// bit.ly/2u3QMZl) per la descrizione dettagliata delle caratteristiche del prototipo.

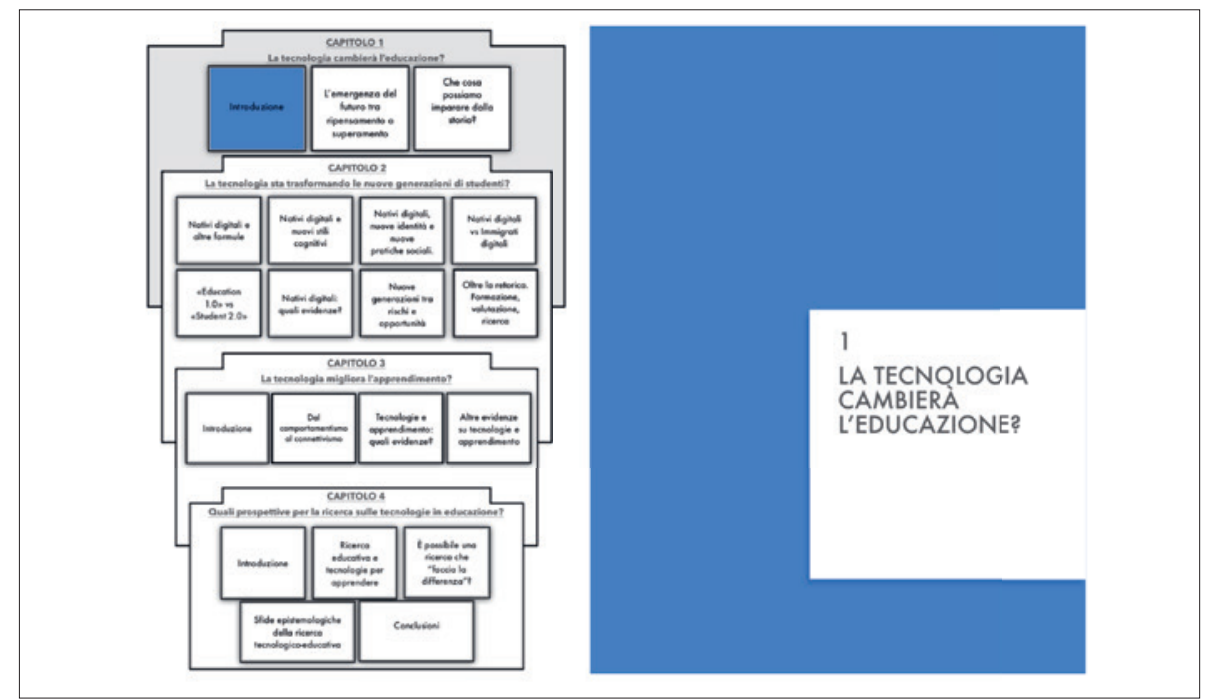

Fig. 1 Visual map interattiva che viene riproposta all'inizio di ogni capitolo e permette la navigazione intuitiva e rapida del libro.

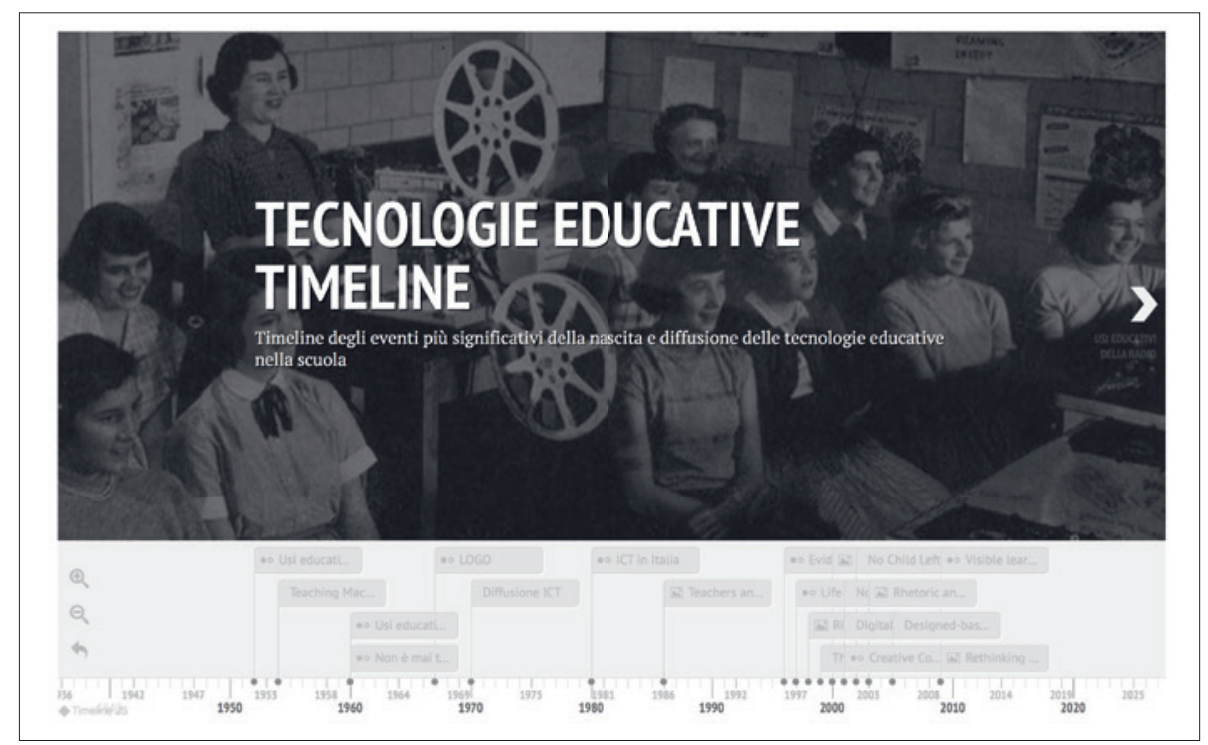

Fig. 2 Timeline interattiva realizzata con «Timeline JS» di Knight Lab che aiuta lo studente nella ricostruzione delle tappe fondamentali della storia delle tecnologie educative. 


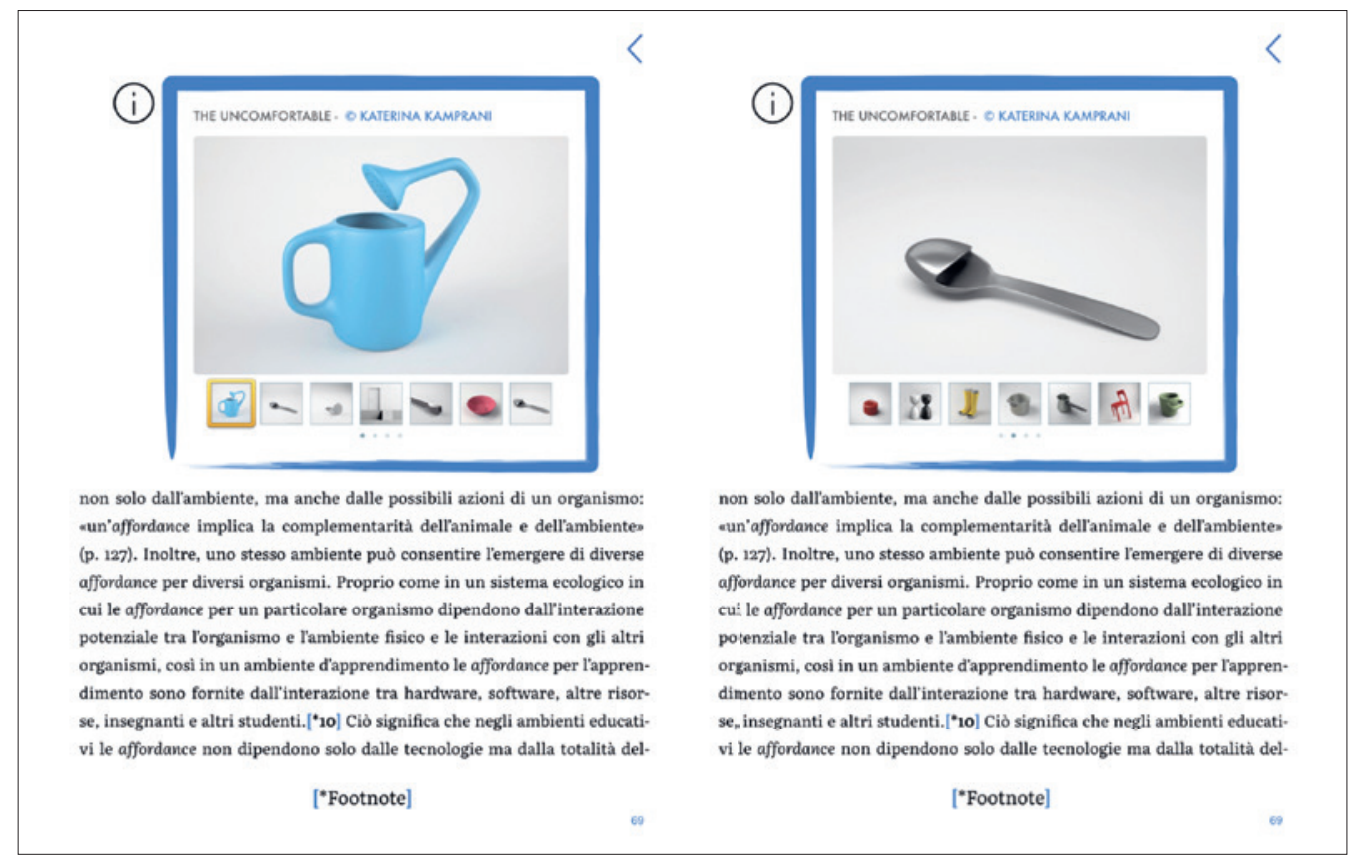

Fig. 3 Galleria fotografica interattiva dedicata alla serie «The Uncomfortable» (https://www.theuncomfortable.com/) della designer Katerina Kamprani utile alla spiegazione del concetto di affordance.

\section{Risultati dello studio di prototipazione}

Sintetizzando i risultati della valutazione su un totale di 12 items di valutazione dell'usabilità dell'interfaccia $58(48.3 \%)$ giudizi sono risultati «molto positivi», $53(44.16 \%)$ «positivi», $8(6.6 \%)$ «intermedi» e soltanto $1(0.83 \%)$ «negativo». Su un totale di 11 item di valutazione dell'efficacia del supporto per l'apprendimento 32 (29.0\%) giudizi sono risultati «molto positivi»; $53(48.18 \%)$ «positivi», 23 (20.9\%) «intermedi» e soltanto 2 (1.81\%) «negativi». Per la consultazione dei risultati relativi ad ogni singolo item e per i grafici di sintesi si rimanda online (http://bit.ly/2KL5HCp), mentre in questa sede commentiamo alcuni dei dati più interessanti alla luce delle scelte di design e delle indicazioni fornite dagli esperti relativamente alla loro «efficacia».

Dalla valutazione è emerso in primo luogo come un libro elettronico di questo tipo, se disegnato seguendo alcune buone regole di design, possa rappresentare un oggetto appropriato al target universitario e un'alternativa appetibile per gli studenti. Per gli esperti l'interfaccia grafica ha infatti un adeguato look and feel, risulta facile da utilizzare e l'organizzazione dello spazio, l'impaginazione dei contenuti e l'utilizzo degli elementi tipografici sono funzionali a migliorare la leggibilità.

Durante la progettazione abbiamo cercato di replicare il più possibile le caratteristiche, il comportamento e l'ergonomia cognitiva della pagina di carta, nel tentativo di venire incontro alle aspettative del lettore e non creare esperienze in conflitto con il suo modello mentale. Abbiamo deciso di utilizzare soltanto tre 
font per garantire uniformità ed evitare fenomeni di disorientamento. In vista di una maggiore accessibilità, per il corpo del testo si è scelto di utilizzare il carattere ad alta leggibilità «Literata» sviluppato specificatamente per la lettura su dispositivi digitali. Per non creare ambiguità gli elementi testuali utilizzano soltanto due colori e le stesse scelte cromatiche sono adottate per elementi simili. Anche le icone, gli elementi multimediali e i widgets sono stati personalizzati rispettando le stesse caratteristiche cromatiche. Abbiamo enfatizzato alcuni concetti chiave attraverso un uso significativo del colore e di box testuali che aiutano il lettore a focalizzare l'attenzione su elementi particolarmente importanti che necessitano di un rinforzo. Il panel ha valutato positivamente queste soluzioni ritenendole utili a migliorare la leggibilità complessiva del prototipo.

Come abbiamo visto leggendo un e-book non è possibile beneficiare dei riferimenti fisici offerti dal libro cartaceo, ed è quindi fondamentale curare tutti gli aspetti di supporto alla navigazione che possono aiutare il lettore a monitorare la propria posizione all'interno del testo. Da questo punto di vista lo strumento integrato di miniature di iBooks risulta particolarmente funzionale, permette di avere una preview del testo e navigare con estrema semplicità: il lettore può sapere in ogni momento in quale parte si trova e in quale relazione quella parte sta con il tutto. Per ottimizzare la navigazione del prototipo abbiamo creato un indice grafico interattivo ad hoc che viene riproposto all'inizio di ogni capitolo grazie al quale è possibile sapere a che punto della lettura ci troviamo e raggiungere rapidamente la parte di testo desiderata. Ogni pagina ha un bottone di ritorno all'indice del relativo capitolo. Dai commenti degli esperti sono emersi alcuni problemi tecnici di navigazione del prototipo che sono stati risolti in fase di re-design. Non sono emersi particolari problemi di disorientamento prodotti dal sistema di navigazione: il libro fornisce per gli esperti un rapido accesso alle informazioni e adeguate funzioni di supporto al lettore, possiede appropriati indizi di interfaccia e un buon livello di prevedibilità.

Come fatto notare da un esperto «la possibilità di tornare a casa e di capire quali siano i link esterni sono aspetti importantissimi sempre». I link e le risorse esterne al testo sono stati resi riconoscibili contrassegnandoli con il colore blu e sottolineando il testo come prevede il modello mentale interiorizzato dagli utenti sul Web. Il prototipo è stato arricchito da parole calde che rimandano alle relative risorse di approfondimento e alle voci del glossario. Le citazioni bibliografiche nel corpo del testo sono collegate al relativo riferimento in bibliografia. È possibile consultare un termine nel glossario, la biografia di uno specifico autore, un riferimento bibliografico, una risorsa online, per poi tornare con un click al punto di partenza.

Come evidenziato tuttavia da un altro esperto "arricchire un ebook fino a farlo diventare un ipertesto è una ipotesi di lavoro interessante, ma il "di più" offerto dall'ipertesto non è dal punto di vista della fruizione e dello studio necessariamente meglio della certezza dei riferimenti fisici offerti dal libro cartaceo». È stato necessario porre quindi particolare attenzione a non creare un «eccesso informativo», dosando con cura gli elementi ipertestuali/multimediali e perfe- 
zionare la navigazione dei contenuti in modo da non creare confusione e senso di smarrimento nel lettore. Allo stesso tempo però, come suggerito da un esperto, il rimando a risorse web rappresenta un valore aggiunto proprio nel momento in cui permette di «arricchire l'esperienza di navigazione» e garantisce quella «liquefazione del testo, ovvero la possibilità di riaggregarne i contenuti secondo logiche di attraversamento diverse, diverse anche da quel che l'autrice pensava». Nonostante Ibooks Author non preveda la possibilità di strutturare percorsi personalizzati per diverse tipologie di lettore, abbiamo tentato di fornire strade alternative di fruizione dei contenuti mediante i seguenti strumenti intertestuali: (i) preview interattive dei contenuti in ingresso a ogni capitolo che riportano una sintesi dei concetti più importanti che saranno affrontati con link e rimandi interni al testo (per l'attivazione delle conoscenze); (ii) glossari e mappe concettuali al termine che riassumono quanto detto e permettono al lettore di tornare velocemente a un concetto o argomento affrontato (per il richiamo delle informazioni prima delle verifiche finali). Il panel ha valutato positivamente questi strumenti e il livello di connettività complessiva dell'ipertesto.

Le funzionalità interattive, i vocabolari integrati, i collegamenti ipertestuali ed intertestuali, le funzionalità di ricerca rappresentano un concreto valore aggiunto del libro elettronico rispetto alla controparte cartacea, ma queste funzionalità devono essere progettate al meglio per essere comprese facilmente ed utilizzate dall'utente. Tra le funzionalità più critiche, e che richiedono un livello avanzato di competenze di programmazione, ci sono quelle che riescono a fornire tipologie diverse di feedback allo studente sia sulle azioni che svolge durante la lettura sia sul proprio livello di apprendimento. I sistemi di scrittura di libri di testo elettronici, compreso Ibooks Author, non prevedono ancora funzionalità integrate di feedback e monitoraggio del lettore per aiutare lo studente a scoprire i progressi fatti, gli errori e le incomprensioni. Nonostante questo la valutazione del feedback fornito dall'interfaccia è risultata buona in particolar modo per le verifiche interattive presenti al termine di ogni capitolo che testano lo studente e registrano le risposte.

Secondo gli esperti il sistema possiede un adeguato livello di approfondimenti e l'utilizzo degli elementi multimediali rispetta i principi basilari della teoria cognitiva dell'apprendimento multimediale (es. coerenza e contiguità). Nonostante si sia scelto di fare un uso moderato, mai decorativo, della multimedialità nel tentativo di evitare il più possibile fenomeni distrattivi, l'impiego di elementi extratestuali non è stato giudicato sempre tale da non creare fenomeni di sovraccarico cognitivo, il che sottolinea ancora una volta l'importanza di calibrare in modo molto attento il loro inserimento.

Anche se l'efficacia del sistema di authoring dipende, in buona parte, non dalla nostra progettazione, quanto dall'efficacia delle funzionalità del dispositivo e dell'interfaccia Apple, il sistema garantisce per gli esperti un adeguato supporto al lettore. Se in genere le applicazioni di lettura digitale risultano ancora carenti per la possibilità di personalizzare l'esperienza di lettura attraverso annotazioni, sottolineature, evidenziazioni, segnalibri, appunti, pratiche comuni durante lo 
studio e la lettura attenta di testo stampato, iBooks sembra non presentare particolari criticità e permette inoltre inedite possibilità di lavoro sul testo, come nel caso delle schede riassuntive per lo studio (flash card) che il sistema genera automaticamente con le parti evidenziate e le annotazioni prese dal lettore e raccoglie in un unico ambiente di revisione.

\section{Limiti dello studio e possibili sviluppi futuri}

Un limite dello studio è rappresentato dal numero esiguo di esperti coinvolti nella valutazione. Purtroppo non è stato possibile intervenire su questo elemento di criticità dovuto principalmente alla scarsa interoperabilità del prototipo.

Tra i possibili sviluppi futuri lo studio potrebbe essere ripetuto coinvolgendo un numero più ampio di esperti di valutatori e prevedendo un sistema di analisi dei logs per capire dove gli esperti abbiano soffermato la loro attenzione per più tempo, di quali loop siano rimasti prigionieri, quali difficoltà abbiamo incontrato nella navigazione. Occorrerebbe inoltre sondare l'aspetto emotivo dell'esperienza, quanto volentieri la ripeterebbero, la consiglierebbero ai colleghi, quanto si siano sentiti immersi nel contenuto del libro e quanto coinvolti nella sua lettura, quali elementi multimediali abbiano apprezzato maggiormente e quali trovato eccessivi.

\section{Discussione}

Ci siamo chiesti come fare a progettare un testo sfruttando alcune prerogative del digitale ed abbiamo sviluppato un prototipo in base alle indicazioni provenienti dalla letteratura di riferimento. La rassegna della letteratura ha evidenziato ancora una certa confusione a livello terminologico, la mancanza di linee guida e modelli di sviluppo condivisi, uno scenario dominato perlopiù dall'iniziativa di singole realtà scolastiche e soggetti del mondo dell'editoria.

I principi e le regole di progettazione reperite in letteratura e applicate al processo di prototipazione del libro di testo digitale sembrano comunque aver prodotto buoni risultati. La valutazione/validazione del libro da parte degli esperti è risultata molto positiva e non sono state riscontrate evidenti criticità. Il processo di progettazione e sviluppo del prototipo è risultato tuttavia più impegnativo del previsto, sia dal punto di vista delle risorse richieste (su tutte il tempo) che dal punto di vista della complessità e dell'eterogeneità degli elementi chiamati in gioco. Allo stesso tempo la progettazione e la realizzazione del prototipo si sono rivelate esperienze estremamente formative che hanno permesso di unire la teoria alla pratica creando tra le due relazioni fortemente sinergiche. La teoria ha regolarmente informato la pratica durante il design del prototipo, così come la realizzazione ha fornito di volta in volta indicazioni per «rivedere» la teoria. L'idea dell'autoproduzione è attraente proprio per la portata altamente formativa che può avere questo processo media-educativo: riflettere e lavorare insieme alla 
realizzazione di oggetti così complessi presenta una quantità di possibili stimoli pedagogici sotto il profilo specificamente editoriale, della conoscenza degli strumenti di programmazione, delle problematiche relative alla privacy, al copyright dei contenuti, dell'organizzazione testuale, delle relazioni tra i vari media, dei linguaggi coinvolti e produce senz'altro benefici sul piano delle conoscenze, ma soprattutto su quelli della riflessività e dell'autoregolazione degli studenti.

\section{Conclusioni}

Nonostante l'entusiasmo di chi immagina docenti e studenti finalmente «liberati» dalle regole imposte da editori e professionisti del settore e un futuro di autoproduzioni, chi intenda avventurarsi nella realizzazione di un libro di testo digitale dovrà necessariamente fare $\mathrm{i}$ conti con il fatto che progettare, scrivere, disegnare un materiale didattico di questo tipo non è opera semplice, né tantomeno rapida. Oramai lo sappiamo, con il digitale le competenze non scompaiono ma si moltiplicano: per la scrittura di un libro elettronico servono non soltanto abilità professionali sotto il profilo specificamente editoriale, ma anche un'adeguata dimestichezza con gli strumenti di programmazione, scrittura ed editing.

Occorre padroneggiare gli strumenti di gestione e pubblicazione dei contenuti, le regole connesse alla privacy e al copyright dei materiali. Organizzare e assemblare in maniera funzionale materiali multicodicali di natura diversa richiede la conoscenza dei principi che regolano i diversi media. Le competenze «tecnologiche» vanno poi coniugate a quelle più strettamente formative. L'insegnante dovrebbe divenire in quest'ottica un vero e proprio Instructional Designer (ID) in grado di orchestrare una serie complessa di elementi. Al momento quindi pensare che i processi di autoproduzione possano essere messi a sistema, se prima non si hanno modelli di riferimento per il design, così come processi strutturati di acquisizione delle competenze necessarie al corpo docente, è sinceramente illusorio (sempre se al di là degli slogan l'obiettivo è quello di produrre contenuti caratterizzati da un certo livello di qualità, complessità, strutturazione, autorevolezza e validazione). ${ }^{8}$

Il digitale possiede degli elementi di innegabile «facilitazione naturale» che possono, sotto certe condizioni, approfondire ed arricchire l'esperienza lettura, spingendo lo studente al superamento di un approccio puramente nozionistico del sapere, e la possibilità di usufruire di alcune caratteristiche del medium digitale può inoltre portare, in certi casi, ad una comprensione più profonda dell'oggetto

\footnotetext{
In attesa che il Ministero fornisca delle linee-guida e regole più specifiche che aiutino e orientino il lavoro di produzione, va in questo senso quanto fatto negli ultimi anni dall'Istituto Nazionale di Documentazione, Innovazione e Ricerca Educativa (INDIRE) che, all'interno del progetto Avanguardie Educative (http://avanguardieeducative.indire.it/), sta collaborando assieme ai docenti di alcune scuole che già sperimentano l'autoproduzione di libri di testo e contenuti didattici digitali, per studiare soluzioni sostenibili e condivise.
} 
di studio. Si deve però rilevare come ancora oggi troppo spesso, per discutere e argomentare sulle prerogative del digitale per l'apprendimento e di opportunità come la reticolarità, l'interattività, la multimedialità, si scelga la fissità e la staticità di testi che, per quanto digitalizzati, altro non sono che .pdf e poco più. In altre parole da una parte si elogiano le proprietà di scardinamento che il digitale avrebbe e dall'altra ci si scontra forse poi con le reali difficoltà (anche puramente tecniche) che questa opera di scardinamento richiederebbe. Non si vuole naturalmente dubitare della sincerità di tali riflessioni, ma mostrare solo ciò che negli ultimi anni ha accompagnato la riflessione sulla lettura digitale: molte ipotesi, riflessioni, previsioni e pochi standard e modelli di riferimento, pochi casi concreti o strade che abbiano convinto più di altre.

I libri di testo digitali possiedono potenzialità innovative delle pratiche didattiche e di studio che non possono essere ignorate, ma in base alla nostra esperienza, se l'obiettivo è quello di ottenere prodotti rigorosi, didatticamente e qualitativamente apprezzabili e validati, abbiamo bisogno di attendere che la ricerca informi maggiormente la pratica e vi siano modelli di riferimento per la progettazione e lo sviluppo dei nuovi testi, così come strumenti, criteri e soggetti predisposti a valutarne la qualità, oltre naturalmente a chi quel materiale lo produce e consigliamo quindi al momento maggiore cautela.

\section{Bibliografia}

Baccino, T., \& Drai-Zerbib, V. (2012). L'usage des tablettes numériques : évaluations ergonomiques. Présenté à Colloque Ecritech 3, Nice, https://bit. ly/2DdelpN

Bastien, J., \& Scapin, D. (1993). Ergonomic criteria for the evaluation of Human Computer interfaces. International Journal of Human-Computer Interaction, 4(2), 183-196.

Bozkurt, A., \& Bozkaya, M. (2015). Evaluation Criteria for Interactive E-Books for Open and Distance Learning. The International Review of Research in Open and Distributed Learning, 16(4), 58-82.

Calvani, A. (2002). Per una «ergonomia didattica».Form@re-Newsletter per la formazione in rete, 13, http://bit.ly/2g7gwgt [Accesso 19.06.2018]

Calvani, A. (2008). Educazione comunicazione e nuovi media: sfide pedagogiche e cyberspazio. Torino: UTET.

Calvani, A. (2013). L'innovazione tecnologica nella scuola: come perseguire un'innovazione tecnologica sostenibile ed efficace. LEA - Lingue e Letterature d'Oriente e d'Occidente, 2, 567-584, http://bit.ly/2hJauGA [Accesso 19.06.2018]

Clark, R.C., \& Lyons, C. (2011). Graphics for Learning: Proven Guidelines for Planning, Designing, and Evaluating Visuals in Training Materials. San Francisco: Pfeiffer. 
Crestani, F., Landoni, M., \& Melucci, M. (2005). Appearance and functionality of electronic books. International Journal on Digital Libraries, 6(2), 192209.

DeStefano, D., \& LeFevre, J. (2007). Cognitive load in hypertext reading: A review. Computers in Human Behavior, 23(3), 1616-1641.

Díaz, P. (2003). Usability of hypermedia educational e-books. D-Lib magazine, 9(3), http://bit.ly/2xO6gUA [Accesso 19.06.2018]

Dix, A., Finlay, J.E., Abowd, G.D., \& Beale, R. (2004). Human-computer interaction (3rd. ed.). Upper Saddle River, NJ: Pearson Education.

Gong, C., Chen, G., Wang, X., Zhang, X., \& Huang, R. (2013). The functions of e-textbooks for utilizing in K-12 classes: A case study in Beijing. Advanced Learning Technologies, 479-480.

Gu, X., Wu, B., \& Xu, X. (2014). Design, development, and learning in e-Textbooks: what we learned and where we are going. Journal of Computers in Education, 2(1), 25-41.

Holsanova, J., Holmberg, N., \& Holmqvist, K. (2009). Reading information graphics: The role of spatial contiguity and dual attentional guidance. Applied Cognitive Psychology, 23(9), 1215-1226.

ISO 9241-110 (2006). Ergonomics of human-system interaction - Part 110: Dialogue principles, http://bit.ly/2xPtQj [Accesso 19.06.2018]

Kalyuga, S., \& Renkl, A. (2010). Expertise reversal effect and its instructional implications: Introduction to the special issue. Instructional Science, 38, 209215.

Landoni, M. (1997). The Visual Book system: a study of the use of the visual rethoric in the design of electronic books. PhD Thesis, Department of Information Science, University of Strathclyde, Glasgow, Scotland, UK.

Landoni, M., Crestani, F., \& Melucci, M. (2000). «The Visual Book and the Hyper-TextBook: Two Electronic Books One Lesson?» RIAO 2000 (Recherche d'Informations Assistee par Ordinateur - «Computer-Assisted Information Retrieval») Conference Proceedings, Paris, April, pp. 247-265.

Landoni, M., Wilson, R. \& Gibb, F. (2000). From the visual book to the WEB Book: the importance of design. The Electronic Library, 18(6), 407-19.

Li, L., Chen, G., \& Yang, S. (2013). Construction of cognitive maps to improve e-book reading and navigation. Computers \& Education, 60(1), 32-39.

MacFadyen, H. (2011). The reader's devices: The affordances of ebook readers. Dalhousie Journal of Interdisciplinary Management, 7, 1-15.

MIUR. Ministero dell'Istruzione, dell'Università e della Ricerca (2012). Decreto Ministeriale 781 del 27 settembre 2013. Libri di testo, https://bit.ly/2LANS54 [Accesso 19.06.2018]

MIUR. Ministero dell'Istruzione, dell'Università e della Ricerca (2013). Legge 128 del 8 novembre 2013 recante misure urgenti in materia di istruzione, università e ricerca, https://bit.ly/2uM7Lix [Accesso 19.06.2018] 
Moreno, R., \& Mayer, R. E. (1999). Cognitive principles of multimedia learning: The role of modality and contiguity. Journal of Educational Psychology, 91, 358-368.

Nielsen, J. (1993). Usability Engineering. Boston: Academic Press.

Nielsen, J. (1998). Jakob Nielsen's Alertbox - «Electronic Books-A Bad Idea», http://bit.ly/2xdpjmS [Accesso 19.06.2018]

Pernice, K. (2017). F-Shaped Pattern of Reading on the Web: Misunderstood, But Still Relevant (Even on Mobile). Nielsen Norman Group, http://bit. ly/1VN4fKS [Accesso 19.06.2018]

Railean, E. A. (2015). Psychological and Pedagogical Considerations in Digital Textbook Use and Development. Hershey, PA: IGI Global.

Roncaglia, G. (2010). La quarta rivoluzione. Sei lezioni sul futuro del libro. Bari: Laterza.

Roncaglia, G. (2013). Creare strati, animare i dati dove vanno gli e-book multimediali. Mondo digitale, 45, 1-14, http://bit.ly/2xdEj9m [Accesso 19.06.2018]

Roncaglia, G. (2018). L'età della frammentazione. Cultura del libro e scuola digitale. Laterza: Roma-Bari.

Scapin, D.L., \& Bastien, J.M.C. (1997). Ergonomic criteria for evaluating the ergonomic quality of interactive systems. Behaviour \& Information Technology, 16(4/5), 220-231.

Shneiderman, B., Plaisant, C., Cohen, M., \& Jacobs, S. (2009). Designing the user interface: Strategies for effective human-computer interaction (5th ed.). Boston, MA, Addison-Wesley.

Sweller, J., van Merrienboer, J.J.G., \& Paas, F. (1998). Cognitive architecture and instructional design. Educational Psychology Review, 10(3), 251-296.

Wertheimer, M. (1923). Untersuchungen zur Lehre von der Gestalt. Psychologische Forschung, 4, 301-350.

Wilson, R., Landoni, M., \& Gibb, F. (2002). Guidelines for designing electronic books. Research and Advanced Technology for Digital Libraries: Proceedings of the 6th European Conference, ECDL'02, September 16-18, 2002, Paris, France (pp. 47-60), http://bit.ly/2hKxwN7 [Accesso 19.06.2018]

Wilson, R. (2003). «The 'look and feel' of an ebook: considerations in interface design», Proceedings of the 2002 ACM Symposium on Applied Computing, Madrid, Spain. ACM Press, New York, NY, 2002. 